\title{
Off-equatorial circular orbits in magnetic fields of compact objects
}

\author{
Zdeněk Stuchlík ${ }^{1}$, Jiří Kováŕr ${ }^{1}$ and Vladimír Karas ${ }^{2}$ \\ ${ }^{1}$ Institute of Physics, Faculty of Philosophy and Science, Silesian University in Opava, \\ Bezručovo nám. 13, CZ-746 01, Opava, Czech Republic \\ email: Jiri.Kovar@fpf.slu.cz \\ ${ }^{2}$ Astronomical Institute, Academy of Sciences, Boční II, CZ-141 31, Prague, Czech Republic \\ email: Vladimir.Karas@cuni.cz
}

\begin{abstract}
We present results of investigation of the off-equatorial circular orbits existence in the vicinity of neutron stars, Schwarzschild black holes with plasma ring, and near Kerr-Newman black holes and naked singularities.
\end{abstract}

Keywords. Black hole physics - stars: neutron - magnetic fields - stellar dynamics

The motivation of our work was the analysis of charged particle motion in a pure dipole magnetic field done by Störmer (1955), the so-called 'classical Störmer problem'. It provides a description leading to the understanding of radiation belts composed of individual ions surrounding Earth and other magnetized planets. This problem was generalized by Dullin et al. (2002) to the dynamics of charged dust grains in planetary magnetospheres, when there are smaller charge to mass ratios, and the planetary gravity and co-rotating electric field play a role. Such studies point out the existence of dust grains stable offequatorial circular orbits of constant radius and latitude, the so-called stable halo orbits. As was shown by Howard et al. (1999), the orbits take place near, e.g., Saturn.

We focused on the halo orbits existence in strong gravitational fields, i.e., in the vicinity of three qualitatively different kinds of compact objects, endowed with a dipole-type magnetic field. These are a neutron star, a Schwarzschild black hole with a current loop, and a Kerr-Newman black hole or naked singularity. The first case corresponds to the original Störmer's problem, but with the strong gravity described within the pseudoNewtonian approach by the Paczyński-Wiita potential. The second case, investigated within the general relativity, may serve as a toy model of the black-hole spacetime with a magnetized accretion disc. In the last case, the solution was found by de Felice (1979) and Calvani et al. (1982). But the authors only simply conclude that the halo orbits do exist in Kerr-Newman spacetimes, regardless of the stability of the orbits and Kerr-Newman black-hole and naked-singularity space-time classes.

Our study (see the paper Kováŕ et al. (2008)) points out the stable halo orbits existence near all of the three studied kinds of compact objects. However, in the case of KerrNewman black hole, the stable halo orbits are of marginal astrophysical importance, being hidden under the inner event horizon. Above the outer event horizon, only unstable halo orbits can appear, while the stable circular orbits are allowed in the equatorial plane only. In principle, as follows from our analysis, stable halo orbits become accessible in the Kerr-Newman naked-singularity spacetimes. On the other hand, in the field of neutron stars or near black holes with current loops, the stable halo orbits can be located outside the body and can be astrophysically relevant. Discussion and searching for the halo orbits were performed in the way of searching for minima of 2-dimensional effective potential 
(a)

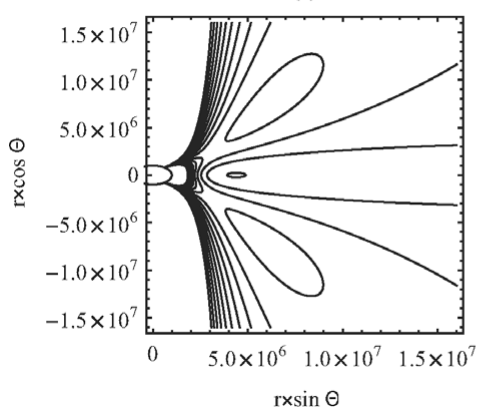

(b)

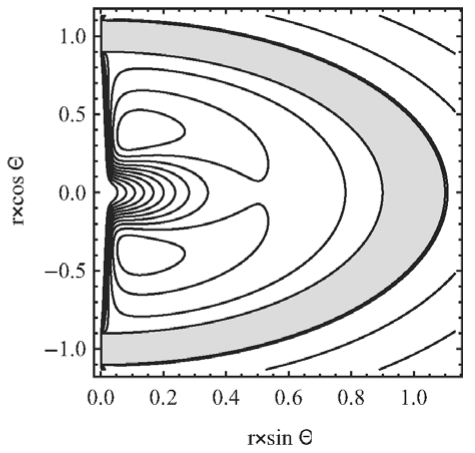

Figure 1. (a) Contours of pseudo-Newtonian effective potential for co-rotating charged dust grains with $q=-10^{-14} \mathrm{C}, m=10^{-17} \mathrm{~kg}$ moving near a neutron star with $\Omega=10^{3} \mathrm{rad} / \mathrm{s}$, $B=10^{8} \mathrm{G}$ and $R=1.2 \times 10^{6} \mathrm{~cm}$. (b) Countours of general relativistic effective potential for motion of charged particles with $\tilde{L}=1$ and $\tilde{q}=-5$ in the Kerr-Newman black-hole spacetime with $a^{2}=0.04$ and $e^{2}=0.95$ (in geometric units and units of $M$ ). The gray region match the black-hole spacetime region between the outer and inner event black-hole horizons; the potential minima correspond to the stable halo orbits positions.

for the charged particles motion and related potential wells, necessary for the charged particles collections (see figure 1).

Halo orbits could have outstanding astrophysical consequences for radiation of accreting compact objects. This is because the halo orbits form two lobes of stable motion - one above and the other one below equatorial plane - through which the accretion disc radiation has to pass on its way towards a distant observer. Therefore, the halo lobes could act as a part of radiation reprocessing corona. The existence of the lobes can have profound consequences even for plasma oscillations. It allows us to imagine a situation where the bulk motion is along the halo orbit in the center of the lobe, around which a fraction of trapped particles oscillate. The oscillations modulate the electromagnetic signal from the plasma cloud, observed as a mode of oscillations in the detected radiation. The halo orbits in the magnetic field of compact stars and black holes could be related to the oscillatory motion with 'halo radial' and 'halo vertical' epicyclic frequencies, which could be considered as a complementary model of the QPOs observed in the binary system of compact stars and microquasars.

Further details can be found in the paper of Kovár et al. (2008).

\section{Acknowledgements}

The work was supported by the Czech GrantsMSM4781305903 and AV0Z10030501.

\section{References}

Calvani, M. et al. 1982, Nuovo Cimento B Serie 67, 1

Dullin, H. R., Horányi, M., \& Howard, J. E. 2002, Physica D 171, 178

de Felice, F. 1979, Physics Letters A 69, 307

Howard, J. E., Horányi M., \& Stewart, G. R. 1999, Physical Review Letters 83, 3993

Kováŕ, J., Stuchlík, Z., \& Karas, V. 2008, Class. Quantum Grav. 25, 095011

Störmer, C. 1955, The Polar Aurora, Clarendon Press, Oxford 